North East and SOUth East thames Regional Health AUTHORTY (1994) The Report of the Inquiry into the Care and Treatment of Christopher Clunis. London: HMSO.

PEAY, J. (1989) Tribunals on Trial: Study of Decision Making under the MHA 1983. Oxford: Clarendon Press.

*Khalida Ismail, Senior Registrar, Maudsley Hospital. London SE5 8AF; Shubulade Smith.
Clinical Research Fellow, Department of Psychological Medicine, Institute of Psychiatry. London and Tony Maden. Senior Lecturer, Department of Forensic Psychiatry. Institute of Psychiatry, London

*Correspondence

\title{
Provision for people with anxiety disorder by a community health care trust
}

\author{
Hugh Middleton, Lisa Ball, David Blore, Helen Dunn, Margaret Foster, \\ Barbara Hart, Ifti Majid and Peter Rajan
}

\begin{abstract}
Aims and method Provision for patients with loosely defined anxiety disorders has been reviewed. During a two-week period, 66 of the 69 adult mental health practitioners working in a modestly sized community health care trust surveyed all patients with anxiety disorder currently under their care.

Results These practitioners were seeing a total of 758 patients who fitted a loose definition of anxiety disorder. A further 134 patients had been treated in anxiety management groups during the preceding year. Disparate treatments were being used, reflecting idiosyncratic approaches to this otherwise homeogenous group of patients.

Clinical Implications Less than one-tenth of the estimated population of anxiety disorder patients were receiving specialised treatment. Among those that were, choices of treatment were arbitrarily determined and idiosyncratic. It was clear that the management of these patients does not fulfill the requirements of 'evidence-based practice'.
\end{abstract}

Recent population-based surveys of psychiatric morbidity (Meltzer et al. 1995) and of the provision of care (Commander et al, 1997) reemphasise established views: that there is widespread morbidity and only a small proportion of it is addressed by practitioners with specialised expertise in this area. Anxiety disorders make a prominent contribution to the sum of psychiatric morbidity. They are common, disabling and not infrequently complicated by the secondary development of other conditions such as substance dependency or depression. Furthermore, they make a considerable contribution to burdens of care upon primary and secondary health services and it has been shown that investment in improving services for patients with panic disorder is economically fruitful (Salvador-Carulla et al, 1995). Against this background we have reviewed one service's provision for people with anxiety disorder in the course of considering how it might be improved.

\section{The study}

Central Nottinghamshire Health Care Trust (CNHCT) is the main provider of psychiatric services across Central Nottinghamshire. The catchment includes a relatively densely populated industrial area, redundant mining villages. an affluent residential centre and farming countryside. Services are divided into four fully integrated teams, each responsible for a sector and working closely with their own consultant psychiatrists. Teams have their own consulting facilities in Mansfield, Sutton-in-Ashfield, Ollerton and Newark-upon-Trent. There are 
Table 1. Numbers, respondents and number of anxiety disorder patients by practitioner grouping

\begin{tabular}{lccc}
\hline Professional background & Number employed & Number responding & $\begin{array}{l}\text { Anxiety disorder } \\
\text { pationts }\end{array}$ \\
\hline $\begin{array}{l}\text { General adult psychiatrists } \\
\text { Clinical psychologists }\end{array}$ & 6 & 6 & 387 \\
$\begin{array}{l}\text { Day hospital: Occupational therapists, registered } \\
\text { mental nurses, art therapists, nurse specialists }\end{array}$ & 26 & 6 & 54 \\
$\begin{array}{l}\text { Community mental health teams: community } \\
\text { psychiatric nurses }\end{array}$ & 29 & 25 & 148 \\
\hline
\end{tabular}

active day hospitals in Newark-upon-Trent and Mansfield.

\section{Procedure}

All mental health practitioners employed by Central Nottinghamshire CNHCT and likely to be involved in the treatment of adults with anxiety disorders were identified. They were invited to take part in the survey by a letter endorsed by the unit general manager. Investigators, who were matched to them by professional background, arranged to obtain information about individual patients on their case list during a two-week 'census window'. This information was acquired by semi-structured interview. In addition details of all anxiety management groups organised by Central Nottinghamshire CNHCT staff during the year (1995) were collated.

\section{Respondents}

Sixty-six practitioners took part in the survey; more than $95 \%$ of those who were eligible. Two clinical psychologists and one art therapist declined to take part. Professional background and seniority of those taking part are given in Table 1.

\section{Methods and measures}

The mean number of individual patients on the case-loads of non-medical community mental health teams members during the 'census fortnight' was 21 , among clinical psychologists it was 35 and among day hospital staff it was 22 , whereas among consultant psychiatrists it was 324. Thus, it was realistic to obtain information about all patients on the case-loads of nonmedical staff but not so for those being seen by psychiatrists. These were surveyed by obtaining information about patients actually seen during the census fortnight and scaling it up accordingly. Practitioners were asked to indicate whether or not patients displayed signs of an anxiety disorder in the form of panic attacks, avoidance, disabling worry or indecision, or ritualised, compulsive behaviour. The presence of one or more of these features in patients who were not also taking routine neuroleptic medication, independent of any other diagnostic descriptor, was considered to be evidence of an anxiety disorder and triggered further enquiries about that patient. Practitioners were asked to identify how frequently and for how long they saw each anxiety disorder patient so identified, what if any other therapeutic input was being provided for that patient, whether or not they felt the patient had further problems. what if any medication was being taken and what form the individual therapy sessions took. Information that was collected about anxiety management groups included the professional background and seniority of group leaders, the average duration and number of sessions, the number of patients so treated and the resources involved in assessment and administration.

\section{Findings}

Individual patients

After scaling up psychiatrists' contributions the survey identified 758 'anxiety disorder' patients among 3326 out- and day patients actively in treatment during the 'census window'. Three hundred and eighty-seven of these were the estimated number being seen by psychiatrists. 54 were being seen by clinical psychologists, 169 by members of the community mental health teams and 148 by members of day hospital staff. These numbers are presented in Table 1. Four hundred and forty-seven (59\%) were female, 432 $(57 \%)$ were taking prescribed medication, 523 (69\%) were considered to have additional problems and $260(34 \%)$ were involved in at least three additional therapeutic activities.

\section{Anxiety management groups}

During 1995 the service ran 22 anxiety management groups in community mental health centres and the day hospital. These involved 134 patients. The mean number of sessions was seven and they had a mean duration of 110 minutes. Each also involved a one hour assessment session for each patient and occupied two practitioners. Drop-out rates varied between 12 and $20 \%$. 


\section{Practitioners}

The 66 respondents were asked to indicate their chosen method of working with anxiety disorder patients. The greater proportion (42) indicated that they would use either eclectic or cognitivebehavioural methods, but more than a third opted for a more esoteric approach (see Table 2). 'Eclectic' was poorly defined and really meant 'whatever seemed appropriate'. Only 14 of the respondents choosing other than an eclectic approach had received any formal psychotherapeutic training over and above that included in their generic professional training.

\section{Comment}

\section{Methodological shortcomings}

The study was an internal investigation conducted without additional resources and therefore simple in design and execution. As a result there are clear methodological shortcomings. Perhaps the three most significant are a difference between the methods of estimating psychiatrists' anxiety disorder case-load and those used for other practitioners, a highly simplified method of identifying patients as suffering from an anxiety disorder and a risk of double registration of patients being seen by more than one practitioner.

More than half the studied population and those among them who were identified as anxiety disorder patients were from psychiatrists' case-loads, and because of their size these were sampled and estimated indirectly in contrast with the smaller number from other types of practitioners' case-loads. It is difficult to see how this might have distorted findings. The sample was all out-patients seen by the psychiatrist or their trainee during a two-week period. Findings from this sample were scaled up proportionally to reflect the full size of the psychiatrist's case-load. There is no reason to believe that out-patients seen during the two-

Table 2. Therapeutic approaches in use in Central Nottinghamshire Community Health Care Trust

\begin{tabular}{ll}
\hline Usual therapeutic approach & Number of practitioners \\
\hline Eclectic & 23 \\
Cognitive/behavioural & 19 \\
Humanistic & 8 \\
Psychodynamic & 7 \\
Supportive & 4 \\
Gestalt & 2 \\
Cognitive/analytical & 2 \\
Family centred & 1 \\
\hline
\end{tabular}

week sampling period were anything other than a random sample of the overall out-patient population. The method of identifying an individual patient as one with an anxiety disorder did not apply more rigorous formal diagnostic criteria and overlooked the presence of other potentially comorbid conditions. The only systematic attempt to overcome this was the exclusion of patients known to be taking longterm neuroleptic medication and therefore quite possibly suffering from a chronic psychosis. This method of identifying anxiety disorder patients, by the presence of cardinal features of an anxiety disorder irrespective of other considerations, was chosen in order to reduce the effect of inter-professional differences in diagnostic criteria. Its effects upon the findings, if any, are likely to have been an inflation of the number of anxiety disorder patients by cases in which a more critical approach would have identified a different axis I diagnosis. Nevertheless even if some of the identified patients were not suffering an anxiety disorder, by narrow definition, all those identified in this way will have been disabled by symptoms (panic, avoidance, worry, rituals) which have been shown to be amenable to cognitivebehavioural therapy. A high proportion of individually treated patients were in receipt of at least one additional therapeutic input. Although attempts were made to restrict registration to only one case-load. inadvertent double registration might also have inflated the number of anxiety disorder patients. Thus the effects of acknowledged methodological shortcomings are likely to be an inflation of the number of anxiety disorder patients. Nevertheless double registration does not invalidate comment upon the level and type of resources going into the treatment of anxiety disorders. which was the main aim of the investigation.

\section{Discussion}

The survey revealed strikingly poor provision for this group of patients. Acknowledged methodological shortcomings are likely to have led to an inflation of the number of anxiety disorder patients under treatment during the census period. Nevertheless the identified 892 is a small proportion of the 9360 predicted by a point prevalence of $3.2 \%$ for all anxiety disorders, and reflects yet again the extent to which psychiatric services reach only a minority of those they might help (Goldberg \& Huxley. 1980).

Where individual patients were enjoying specialised attention, a wide range of treatment approaches were in use and a high proportion of patients were considered to have additional 
problems and were involved in additional therapeutic activities. These findings suggest that either a significant proportion of those who were being treated and were identified as an anxiety disorder patient by the survey were not in fact considered to be so by their therapist, and were being treated primarily for something else, or that treatments intended for individuals with anxiety disorder were less focused and specific as research findings suggest they should be (Barlow \& Lehman, 1996).

As far as anxiety management groups were concerned, these accommodated only a small proportion of the total number of identified anxiety disorder patients and may have been an inefficient use of therapists' time. When assessment time, administration and the fact that groups involved two practitioners working together were taken into account, they actually used some eight hours of practitioner time per patient, time that might have been better used in more individualised sessions.

These conclusions should not be read as criticism of the particular service we have investigated. Although other similar investigations are needed to confirm it, our view is that this was a representative service and comparable provisions elsewhere are unlikely to be significantly different. Instead they point to the continuing shortcomings of our approach to a common, frequently trivialised though often very disabling set of conditions for which effective treatments exist but are only sparsely available.

\section{References}

BARLOW, D. H. \& LeHMAN, C. L. (1996) Advances in the psychosocial treatment of anxiety disorders. Archives of General Psychiatry, 63, 727-735.

COMmander. M. J., SAShidharan. S. P., Odell. S. M.. et al (1997) Access to mental health care in an inner-city health district. I: Pathways into and within specialist psychiatric services. British Journal of Psychiatry. 170. 312-316.

GoldBerG, D. P. \& HuXley, P. (1980) Mental Illness in the Community: The Pathway to Psychiatric Care. London: Tavistock.

Meltzer, H., GiLl. B.. PetTiCrew, M.. et al (1995) The Prevalence of Psychiatric Morbidity among Adults Living in Private Households. London: HMSO.

Salvador-Carulla, L., Segui, J., Fernández-Cano, P., et al (1995) Costs and offset effect in panic disorders. British Journal of Psychiatry. 166 (suppl. 27). 23-28.

*Hugh Middleton, Senior Lecturer, Nottingham University Department of Psychiatry. Duncan Macmillan House, Porchester Road. Nottingham NG3 6AA; Lisa Ball, Psychology Assistant; David Blore, Nurse Specialist, Cognitive/ Behavioural Psychotherapy: Helen Dunn, Senior Occupational Therapist: Margaret Foster, Community Mental Health Team Leader: Barbara Hart, Senior Occupational Therapist: Ifti Majid, Day Hospital Manager and Peter Rajan, Director of Psychology Services, Central Nottinghamshire Community Health Care Trust, Forest Hospital, Mansfield

*Correspondence

\title{
Old age psychiatrists' views on continuing in-patient care
}

\author{
John Wattis, Andrew Macdonald and Paul Newton
} Aims and methods The aim of this study was to elicit
views from UK consultants in old age psychiatry
concerning changes that were taking place in long-
term care for older people with mental illness
(especially dementia) and their views on a draft
consensus statement produced by the Faculty for
Psychiatry of Old Age on behalf of the College. A
postal questionnaire was circulated as part of a wider
survey of 472 consultants and a single postal reminder
was sent to non-responders.
Results Two hundred and forty-two (51\%) consultants
responded. Nearly nine out of 10 respondents were in

favour of continuing NHS consultant-supervised longstay beds and three-quarters of them preferred a return to national numerical guidelines for bed numbers, though at a level approximately half of previous guidelines and provision. This was accompanied by a view that such beds should no longer be 'for life' with over three-quarters of respondents supporting discharge if, for example, behaviour problems resolved. If Government policy continued to support 'eligibility criteria' rather than guidelines, $60 \%$ were in favour of national rather than local criteria. There was very strong support for the draft consensus statement (now 